\title{
THE MASSES OF THE MILLISECOND PULSAR J1012+5307 AND ITS WHITE DWARF COMPANION ${ }^{1}$
}

\author{
M. H. VAN KerkwiJK, ${ }^{2,3}$ P. Bergeron, ${ }^{4}$ AND S. R. KulKarnI ${ }^{2}$ \\ Received 1996 May 2; accepted 1996 June 7
}

\begin{abstract}
We report on spectroscopy of the white dwarf companion of the millisecond radio pulsar PSR J1012+5307. We find strong Balmer absorption lines, as would be expected for a cool DA white dwarf. The profiles are much narrower than usual, however, and lines are seen up to H12, indicating that the companion has a low gravity and hence a low mass. This is consistent with the expectation-based on evolutionary considerations and on the mass function - that it is a low-mass white dwarf with a helium core. By comparing the spectra to model atmospheres, we derive an effective temperature $T_{\text {eff }}=8550 \pm 25 \mathrm{~K}$ and a surface gravity $\log g=6.75 \pm 0.07$ cgs. Using the Hamada-Salpeter mass-radius relation for helium white dwarfs, with an approximate correction for finitetemperature effects, we infer a mass $M_{\mathrm{WD}}=0.16 \pm 0.02 M_{\odot}$. This is the lowest mass among all spectroscopically identified white dwarfs. We determine radial velocities from our spectra, and we find a radial-velocity amplitude of $280 \pm 15 \mathrm{~km} \mathrm{~s}^{-1}$. With the pulsar's radial-velocity amplitude, the mass ratio $M_{\mathrm{PSR}} / M_{\mathrm{WD}}=13.3 \pm 0.7$. From all constraints, we find that with $95 \%$ confidence, $1.5<M_{\mathrm{PSR}} / M_{\odot}<3.2$.
\end{abstract}

Subject headings: binaries: close — pulsars: individual (PSR J1012+5307) — stars: neutron — white dwarfs

\section{INTRODUCTION}

Knowledge of the properties of the white dwarf companions of radio pulsars can provide unique constraints on the characteristics and evolution of these binaries, as well as on those of its constituents. Of particular interest are mass determinations for millisecond pulsars. Evolutionary models indicate that millisecond pulsars have accreted up to $0.7 M_{\odot}$ (e.g., van den Heuvel \& Bitzaraki 1995). Hence, if the neutron star started with the "canonical" $1.4 M_{\odot}$ (e.g., Timmes, Woosley, \& Weaver 1996; see, for a recent census, van Kerkwijk, van Paradijs, \& Zuiderwijk 1995), it would now be $\gtrsim 2 M_{\odot}$. If so, this would strongly favor a stiff equation of state (EOS) at supranuclear density (e.g., Cook, Shapiro, \& Teukolsky 1994). For softer EOS, like the one recently proposed by Brown \& Bethe (1994), such a massive neutron star would collapse into a black hole. Thus far, constraints on the masses could only be set from Shapiro delay of the pulsed signal. As this requires (rare) fortuitous alignment, a reasonable constraint is available for one system only, PSR B1855+09, for which Kaspi, Taylor, \& Ryba (1994) found $M_{\mathrm{PSR}}=1.50_{-0.14}^{+0.26} M_{\odot}$.

The temperature of the white dwarf-combined with the mass-can be used to determine the age from theoretical cooling tracks. The cooling age gives a limit to the time since mass transfer to the neutron star ceased. With mass estimates from the mass function and approximate cooling models, such limits have been used to show that magnetic fields in neutron stars do not decay on a $\sim 10^{7}$ yr timescale (Kulkarni 1986), as had been argued previously (see, for a review, Phinney \& Kulkarni 1994). Recently, Bell et al. (1995) used a lower limit to the cooling age of the white dwarf companion of PSR J0034-0534 to infer that the pulsar's initial spin period was $\lesssim 0.6 \mathrm{~ms}$. If so, this would also constrain the structure of

\footnotetext{
${ }^{1}$ Based on observations obtained at the W. M. Keck Observatory on Mauna Kea, Hawaii, which is operated jointly by the California Institute of Technology and the University of California.

2 Palomar Observatory, California Institute of Technology 105-24, Pasadena, CA 91125; mhvk@astro.caltech.edu and srk@astro.caltech.edu.

${ }^{3}$ Hubble Fellow.

${ }^{4}$ Département de Physique, Université de Montréal, C. P. 6128, Succ. Centre-Ville, Montréal, Québec, Canada H3C 3J7; bergeron@astro.umontreal.ca.
}

neutron stars, excluding the stiffer EOS and thus making it hard to have masses $\gtrsim 2 M_{\odot}$ (Cook et al. 1994; Haensel, Salgado, \& Bonazzola 1995). Without accurate white dwarf masses and good cooling models, however, such arguments remain inconclusive.

Masses and temperatures of DA white dwarfs can be determined accurately from their spectra (e.g., Bergeron, Saffer, \& Liebert 1992), and, therefore, we have started a spectroscopic investigation of all bright companions (see van Kerkwijk 1996; currently, six companions with $V \lesssim 23$ are known). First, results can be found in van Kerkwijk \& Kulkarni (1995).

Here we describe spectroscopy of the white dwarf companion of the millisecond pulsar PSR J1012+5307, discovered recently by Nicastro et al. (1995). The system's orbital period is $14.5 \mathrm{hr}$, and the mass function indicates a low-companion mass: $M_{\mathrm{WD}}=0.11 M_{\odot}$ for $M_{\mathrm{PSR}}=1.4 M_{\odot}$ and inclination $i=90^{\circ}$. At the pulsar's position, Nicastro et al. found a faint star on the Palomar Sky Survey. Lorimer et al. (1995) confirmed the positional coincidence, and they found that the star had magnitudes and colors consistent with those of a low-mass white dwarf at the distance implied by the pulsar dispersion measure $(\sim 0.5 \mathrm{kpc})$.

\section{OBSERVATIONS}

Spectra of the companion of PSR $\mathrm{J} 1012+5307(V=19.5$ mag) were taken at the Keck $10 \mathrm{~m}$ telescope with the low-resolution imaging spectrometer (LRIS). On 1995 June 4, the 300 line $\mathrm{mm}^{-1}$ grating was used for a classification spectrum, covering the wavelength range of $3430-8460 \AA$ at $2.5 \AA$ pixels ${ }^{-1}$. With a slit width of $1^{\prime \prime}$, the resolution was $\sim 12$ $\AA$. The conditions were photometric, and for calibration an exposure was taken through a $4 "$ slit. Directly following the object, the spectrophotometric standard Feige 34 (sdO, $V=11.23$, Massey et al. 1988) was observed through both slits, at very similar airmass.

On 1995 November 22-25 and 1996 January 21, the 600 line $\mathrm{mm}^{-1}$ grating and 0.7 slit were used to increase the resolution to $\sim 4 \AA$. The wavelength range covered was $3500-6000 \AA$. 

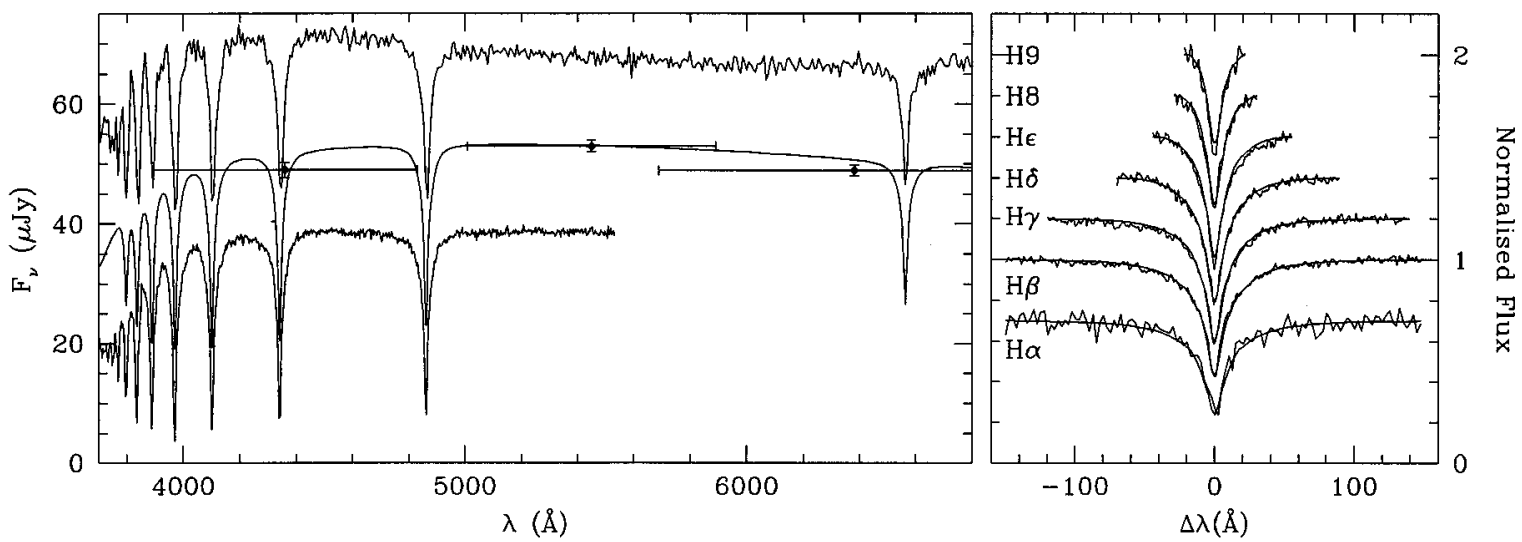

FIG. 1.-Spectrum of the companion of PSR J1012+5307. Shown in the left-hand panel are the spectra taken with the 300 line $\mathrm{mm}^{-1}$ grating (top curve) and the average of the spectra taken with the 600 line $\mathrm{mm}^{-1}$ grating (bottom curve; the spectra were shifted to zero velocity before averaging). The two were normalized to the observed $V$-band flux $(V=19.58$; Lorimer et al. 1995), and are shown offset by +15 and $-15 \mu \mathrm{Jy}$, respectively. Also shown are the observed broadband fluxes and the best-fit pure hydrogen model spectrum. The latter was derived from a fit to the profiles of $\mathrm{H} \beta$ up to $\mathrm{H} 8$, and has $T_{\text {eff }}=8550 \pm 25 \mathrm{~K}$ and $\log g=6.75 \pm 0.07$. In the right-hand panel, the observed line profiles, including those of $\mathrm{H} \alpha$ and $\mathrm{H} 9$, are shown with the modeled ones superposed.

During these runs, the conditions were not photometric, but Feige 34 was again observed, in order to obtain relative spectrophotometry and to verify the stability of the velocity determinations.

The reduction of all spectra was done using MIDAS ${ }^{5}$ and programs running in the MIDAS environment. The frames were bias-corrected, flat-fielded and sky-subtracted using standard procedures, and the spectra was extracted using optimal weighting. Fluxes were determined by first calibrating the wide-slit exposure relative to that of Feige 34 (correcting for small differences in airmass using the extinction curve of Beland, Boulade, \& Davidge 1988), and then by scaling the narrow-slit exposure in such a way that it had the same continuum flux.

\section{EFFECTIVE TEMPERATURE, SURFACE GRAVITY, AND MASS}

The spectra of the companion of PSR J1012+5307 are shown in Figure 1. Strong Balmer lines are seen, from $\mathrm{H} \alpha$ up to H12. In field white dwarfs, the lines are usually broader, and the higher members of the Balmer series are rarely seen, since the corresponding excitation levels are quenched by the high pressure. This indicates that the white dwarf has a low surface gravity and, therefore, a low mass.

The line profiles could be reproduced well with a white dwarf model atmosphere (Fig. 1), with an effective temperature $T_{\text {eff }}=8550 \pm 25 \mathrm{~K}$ and a surface gravity $\log g=$ $6.75 \pm 0.07$ cgs. (For details about the procedure, see Bergeron et al. 1992.) By way of verification, we also fitted the broadband fluxes, determined by Lorimer et al. (1995), to a model atmosphere, with the surface gravity fixed to $\log g=6.75$. We find $T_{\text {eff }}=8730 \pm 110 \mathrm{~K}$, which is consistent with the spectroscopic determination.

The mass of the white dwarf can be estimated from the surface gravity using a mass-radius relation appropriate for a white dwarf with a helium core (given the low mass, the star will never have burnt helium). Unfortunately, helium white dwarfs have not been studied as well as the usual carbonoxygen white dwarfs, and only the zero-temperature massradius relation of Hamada \& Salpeter (1961) is available

5 The Munich Image Data Analysis System is developed and maintained by the European Southern Observatory.
(Table 1). At $8500 \mathrm{~K}$, however, a white dwarf with a $\log g$ as low as that for our white dwarf will not have reached its zero-temperature configuration. To estimate the relative "puffiness," we have used the Wood (1995) models for white dwarfs with pure carbon cores and thick hydrogen atmospheres (Table 1). Inter- and extra-polating in the corrected mass-radius relation, our final estimate for the mass of the white dwarf is $\log M_{\mathrm{wD}} / M_{\odot}=-0.78 \pm 0.06$ (where the quoted uncertainty combines in quadrature an uncertainty of 0.03 because of the uncertainty in $\log g$, and an estimated 0.05 because of the uncertainty in the mass-radius relation). Hence, $M_{\mathrm{WD}}=0.16 \pm 0.02 M_{\odot}$.

\section{RADIAL VELOCITIES AND THE MASS OF THE NEUTRON STAR}

We determined radial velocities for both the companion of PSR J1012+5307 and Feige 34, by fitting the $\mathrm{H} \beta, \mathrm{H} \gamma$, and $\mathrm{H} \delta$ lines simultaneously to Lorentzian profiles, with the widths and velocities of the three lines forced to be the same. By way of verification, we also made fits with the velocities left free. The results were consistent with each other for all spectra except those taken with the 300 line $\mathrm{mm}^{-1}$ grating. For Feige 34 , we found that the velocity derived from $\mathrm{H} \alpha$ was consistent

TABLE 1

Mass-Radius Relation Including Finite-Temperature EFFECTS

\begin{tabular}{ccccc}
\hline \hline $\begin{array}{c}M \\
\left(M_{\odot}\right)\end{array}$ & $\begin{array}{c}R_{0} \\
\left(R_{\odot}\right)\end{array}$ & $\begin{array}{c}\log g_{0} \\
\left(\mathrm{~cm} \mathrm{~s}^{-2}\right)\end{array}$ & $R_{8500} / R_{0}$ & $\begin{array}{c}\log g \\
\left(\mathrm{~cm} \mathrm{~s}^{-2}\right)\end{array}$ \\
\hline $0.154 \ldots \ldots \ldots \ldots$ & 0.0218 & 6.95 & 1.351 & 6.69 \\
$0.213 \ldots \ldots \ldots \ldots$ & 0.0195 & 7.19 & 1.236 & 7.00 \\
$0.305 \ldots \ldots \ldots \ldots$ & 0.0171 & 7.46 & 1.111 & 7.36 \\
$0.399 \ldots \ldots \ldots \ldots$ & 0.0152 & 7.67 & 1.071 & 7.62 \\
$0.499 \ldots \ldots \ldots$. & 0.0137 & 7.86 & 1.048 & 7.82 \\
$0.609 \ldots \ldots \ldots \ldots$ & 0.0122 & 8.05 & 1.032 & 8.02 \\
$0.734 \ldots \ldots \ldots \ldots$ & 0.0108 & 8.24 & 1.022 & 8.22 \\
$0.885 \ldots \ldots \ldots \ldots$ & 0.0092 & 8.46 & 1.010 & 8.45 \\
\hline
\end{tabular}

NoTE.-The first two columns give the zero-temperature mass-radius relation of Hamada \& Salpeter 1961 for a helium white dwarf. The fourth column lists the adopted ratio of the radius at $T=8500 \mathrm{~K}$ and the zero-temperature radius, as determined from the cooling curves of Wood 1995 for carbon white dwarfs with thick hydrogen envelopes. 
TABLE 2

Radial Velocity Measurements

\begin{tabular}{|c|c|c|c|c|}
\hline \multicolumn{3}{|c|}{ PSR J1012+5307 } & \multicolumn{2}{|c|}{ FEIGE 34} \\
\hline $\begin{array}{c}\mathrm{JD}_{\mathrm{bar}} \\
-2450000\end{array}$ & $\phi_{\text {orb }}{ }^{\mathrm{a}}$ & $\begin{array}{c}v \\
\left(\mathrm{~km} \mathrm{~s}^{-1}\right)\end{array}$ & $\begin{array}{c}\mathrm{JD}_{\mathrm{bar}} \\
-2450000\end{array}$ & $\begin{array}{c}v \\
\left(\mathrm{~km} \mathrm{~s}^{-1}\right)\end{array}$ \\
\hline$-127.199 \ldots \ldots$ & 0.027 & $-282 \pm 27$ & -127.184 & $43 \pm 10$ \\
\hline $44.040 \ldots \ldots$ & 0.220 & $-91 \pm 11$ & 44.058 & $40 \pm 4$ \\
\hline $44.150 \ldots \ldots$ & 0.401 & $194 \pm 12$ & 44.161 & $20 \pm 6$ \\
\hline $46.131 \ldots \ldots$ & 0.678 & $74 \pm 19$ & 46.156 & $20 \pm 6$ \\
\hline $46.146 \ldots \ldots$ & 0.704 & $43 \pm 10$ & & \\
\hline $47.097 \ldots \ldots$ & 0.275 & $-19 \pm 19$ & 47.110 & $13 \pm 4$ \\
\hline $103.989 \ldots \ldots$ & 0.362 & $138 \pm 7$ & 103.973 & $7 \pm 4$ \\
\hline $104.014 \ldots \ldots$ & 0.404 & $172 \pm 7$ & & \\
\hline
\end{tabular}

${ }^{\text {a }}$ Using the ephemeris of Lorimer et al. 1995: $T_{0}=\mathrm{JD}_{\text {bar }}$ $2449220.947499(1), P_{\text {orb }}=0.604672713(5)$ days.

with the results from the other spectra, while $\mathrm{H} \beta, \mathrm{H} \gamma$, and $\mathrm{H} \delta$ were off by $60-100 \mathrm{~km} \mathrm{~s}^{-1}$. Therefore, we used the velocity from $\mathrm{H} \alpha$ alone for $\mathrm{J} 1012+5307$ as well.

The velocities were corrected for possible small wavelength calibration errors using the $\mathrm{O}_{\mathrm{I}} \lambda 5577$ night-sky (emission) line. The resulting velocities, corrected to the solar system barycenter, are listed in Table 2, and shown in Figure 2.

The radial velocities were fitted to a circular orbit, with the period and time of ascending node fixed to the radio timing values of Lorimer et al. (1995). We find a radial-velocity amplitude $K_{\mathrm{WD}}=265 \pm 9 \mathrm{~km} \mathrm{~s}^{-1}$ and a systemic velocity $\gamma=-38 \pm 6 \mathrm{~km} \mathrm{~s}^{-1}\left(\chi_{\text {red }}^{2}=0.97\right.$ for 6 dof $)$. If we ignore the uncertain velocity from the low-resolution spectrum, $K_{\mathrm{WD}}=269 \pm 11 \mathrm{~km} \mathrm{~s}^{-1}$ and $\gamma=-40 \pm 7 \mathrm{~km} \mathrm{~s}^{-1}\left(\chi_{\text {red }}^{2}=1.06\right.$ for 5 dof).

The velocities determined for Feige 34 are, on average, more or less consistent with the determinations by Greenstein \& Trimble $\left(1967 ; 17 \pm 13 \mathrm{~km} \mathrm{~s}^{-1}\right)$ and Thejll, MacDonald, \& Saffer (1995; $\left.3 \pm 4 \mathrm{~km} \mathrm{~s}^{-1}\right)$. The individual velocities deviate significantly from the average, however, indicating that some

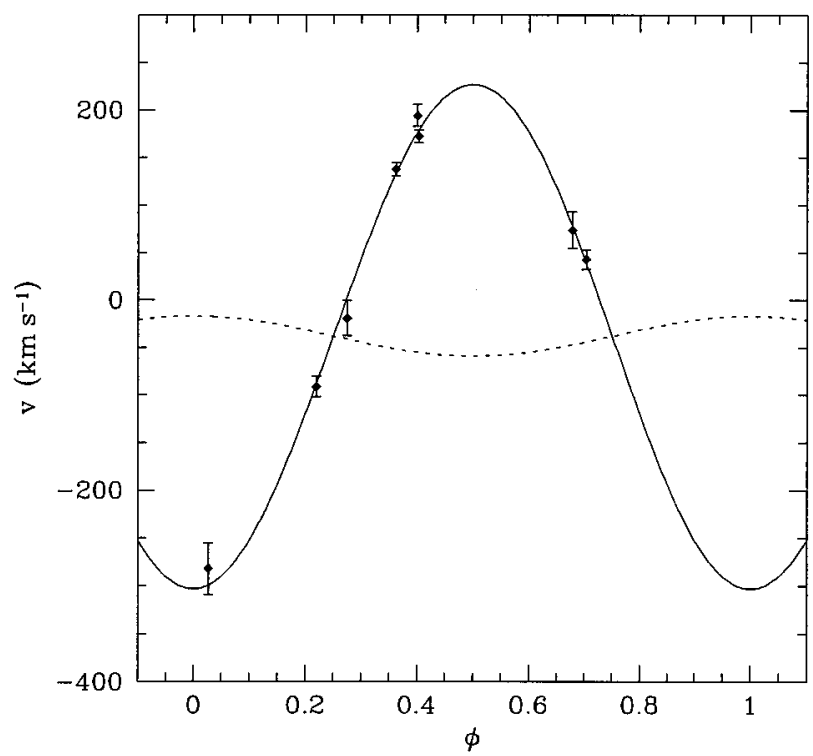

FIG. 2.-Radial-velocity measurements for the companion of PSR $\mathrm{J} 1012+5307$. Overdrawn is the best-fit circular orbit, with the time of ascending node and period fixed to the values determined from the radio timing solution (Lorimer et al. 1995). The radial-velocity curve for the radio pulsar is also shown (dotted curve).

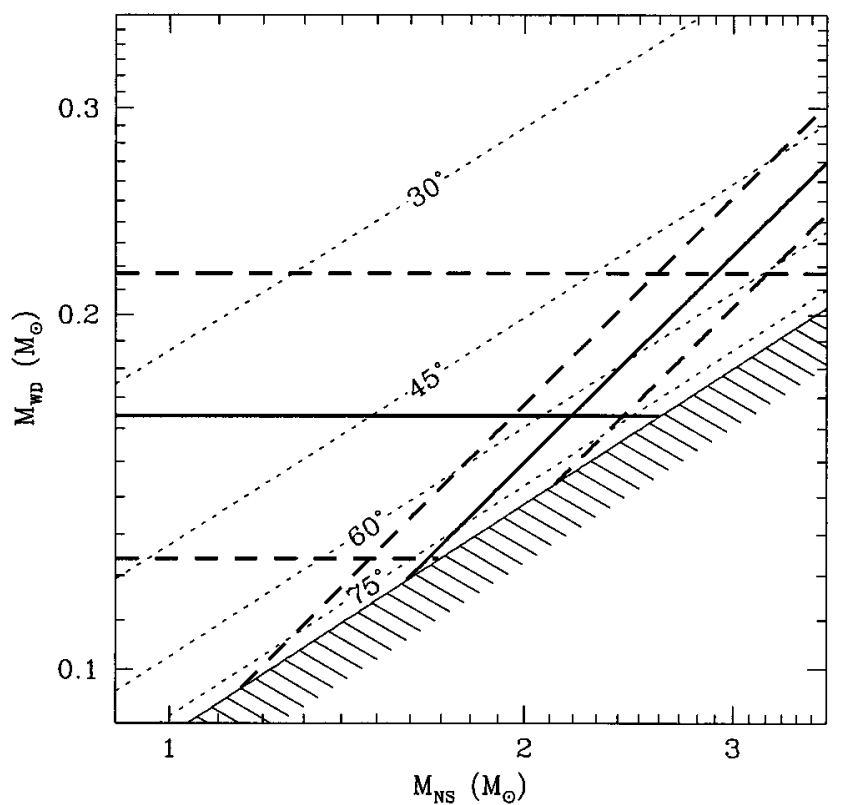

FIG. 3.-Masses of PSR J1012+5307 and its companion. Shown are the constraints from the pulsar mass function (thin, drawn curve for $i=90^{\circ}$; dotted curves for inclinations as indicated), the ratio of the radial-velocity amplitudes (thick, slanted curve), and the white dwarf surface gravity (thick, horizontal curve). For the latter two, the $95 \%$ uncertainty regions are indicated by thick, dashed curves.

systematic effects may be present. To estimate their effect on the orbital solution, we corrected the velocities for $\mathrm{J} 1012+5307$ with those for Feige 34 (corrected for an assumed velocity for Feige 34 of $5 \mathrm{~km} \mathrm{~s}^{-1}$ ), and we refitted the orbit. We find $K_{\mathrm{WD}}=290 \pm 9 \mathrm{~km} \mathrm{~s}^{-1}$ and $\gamma=-62 \pm 9 \mathrm{~km} \mathrm{~s}^{-1}$, with $\chi_{\text {red }}^{2}=0.73$ (ignoring the low-resolution point: $297 \pm 9$, $-66 \pm 9$, and 0.61, respectively). Here the uncertainty in $\gamma$ includes a $5 \mathrm{~km} \mathrm{~s}^{-1}$ uncertainty in the velocity of Feige 34 . The $\chi_{\text {red }}^{2}$ is lower than for the uncorrected velocities, partly because the errors on the velocities now include the uncertainty in the velocities derived for Feige 34. Even if we neglect the latter, however, $\chi_{\text {red }}^{2}$ is still slightly smaller. At present, it does not seem possible to decide which method provides the correct answer, and, therefore, we will conservatively adopt $K_{\mathrm{WD}}=280 \pm 15 \mathrm{~km} \mathrm{~s}^{-1}$ and $\gamma=-50 \pm 15 \mathrm{~km} \mathrm{~s}^{-1}$.

From the radial-velocity amplitudes of the white dwarf and the pulsar [20.9774(2) $\mathrm{km} \mathrm{s}^{-1}$; Lorimer et al. 1995], it follows that the mass ratio $M_{\mathrm{PSR}} / M_{\mathrm{WD}}=13.3 \pm 0.7$. In Figure 3 , we show this constraint, as well as the constraints derived from the pulsar mass function [5.78361(10) $10^{-4} M_{\odot}$ ] and from the white dwarf mass. From this figure, it follows that with $95 \%$ confidence, the mass of the neutron star is within $1.5<$ $M_{\mathrm{PSR}} / M_{\odot}<3.2$.

\section{DISCUSSION AND CONCLUSIONS}

At present, the neutron-star mass determination is not accurate enough to constrain the equation of state, or to test the prediction that a large amount of mass has been accreted. To improve the accuracy, the uncertainties in both the mass ratio and the white dwarf mass need to be reduced. The former will be relatively easy (and may well be interesting on its own; see Fig. 3), and we are planning more precise radial-velocity measurements for this purpose.

It will be less straightforward to improve the white dwarf 
mass determination. Although better models are becoming available (Hansen \& Phinney 1996), there are potential problems related to the uncertainty in the thickness of the hydrogen layer and the unknown abundance of helium in the atmosphere. Helium can be dredged up by convection if the hydrogen layer is thin enough, and, if present, would mimic an increased surface gravity (Bergeron, Wesemael, \& Fontaine 1991). Indeed, Reid (1996) finds that his mass determinations based on the observations of the Einstein redshift are systematically lower than the spectroscopic estimates for temperatures $\lesssim 12,000 \mathrm{~K}$, which could be attributed to the presence of helium. IR observations could potentially resolve this issue (Bergeron, Saumon, \& Wesemael 1995).

If an accurate distance could be determined (via timing parallax or otherwise), it could be combined with the flux and temperature of the white dwarf to yield a precise radius. If different from the radius predicted from our $\log g$ $\left(0.028 \pm 0.002 R_{\odot}\right)$, one could assume either that the problem lies with the mass-radius relation (and not with helium pollution) - and infer a mass from the radius and $\log g$ - or that there is helium pollution, but that the mass-radius relation is fine-and use that to derive a mass from the radius.

From Figure 3 , one sees that the inclination $i \gtrsim 60^{\circ}$ for $M_{\mathrm{PSR}} \lesssim 2 M_{\odot}$. This raises the possibility of a model-independent, accurate determination of the white dwarf mass and the inclination via Shapiro delay. With our precise mass ratio, this would allow one to determine the neutron-star mass accurately.

Finally, there is a small but measurable amount of light that the white dwarf will radiate due to reprocessing of the pulsar wind at its atmosphere. This may provide yet another constraint on the geometry of the system. Fortunately, PSR $\mathrm{J} 1012+507$ is bright in the radio and the companion is relatively bright in the optical, so that all these observations are possible.

We thank Brad Hansen, Yanqin Wu, Fernando Camilo, and Andrew Lyne for useful discussions. M. H. v. K. acknowledges a NASA Hubble Fellowship, and S. R. K. acknowledges grants from NSF, NASA, and the Packard Foundation.

\section{REFERENCES}

Beland, S., Boulade, O., \& Davidge, T. 1988, Canada-France-Hawaii Telescope Info. Bull., 19, 16

Bell, J. F., Bailes, M., Kulkarni, S. R., Leitch, E. M., \& Lyne, A. G. 1995, ApJ, 452, L121

Bergeron, P., Saffer, R. A., \& Liebert, J. 1992, ApJ, 394, 228

Bergeron, P., Saumon, D., \& Wesemael, F. 1995, ApJ, 443, 764

Bergeron, P., Wesemael, F., \& Fontaine, G. 1991, ApJ, 367, 253

Brown, G. E., \& Bethe, H. A. 1994, ApJ, 423, 659

Cook, G. B., Shapiro, S. L., \& Teukolsky, S. A. 1994, ApJ, 424, 823

Greenstein, J. L., \& Trimble, V. L. 1967, ApJ, 149, 283

Haensel, P., Salgado, M., \& Bonazzola, S. 1995, A\&A, 296, 745

Hamada, T., \& Salpeter, E. E. 1961, ApJ, 134, 683

Hansen, B., \& Phinney, E. S. 1996, in preparation

Kaspi, V. M., Taylor, J. H., \& Ryba, M. F. ApJ, 428, 713

Kulkarni, S. R. 1986, ApJ, 306, L85
Lorimer, D. R., Lyne, A. G., Festin, L., \& Nicastro, L. 1995, Nature, 376, 393 Massey, P., Strobel, K., Barnes, J. V., \& Anderson, E. 1988, ApJ, 328, 315 Nicastro, L., Lyne, A. G., Lorimer, D. R., Harrison, P. A., Bailes, M., \& Skidmore, B. D. 1995, MNRAS, 273, L68

Phinney, E. S., \& Kulkarni, S. R. 1994, ARA\&A, 32, 591

Reid, I. N. 1996, AJ, 111, 2000

Thejll, P., MacDonald, J., \& Saffer, R. 1991, A\&A, 248, 448

Timmes, F. X., Woosley, S. E., \& Weaver, T. A. 1996, ApJ, 457, 834

van den Heuvel, E. P. J., \& Bitzaraki, O. 1995, A\&A, 297, L41

van Kerkwijk, M. H. 1996, in Proc. IAU Coll. 160, ASP Conf. Ser., ed. S.

Johnston, M. Walker, \& M. Bailes (San Francisco: ASP), in press

van Kerkwijk, M. H., \& Kulkarni, S. R. 1995, ApJ, 454, L141

van Kerkwijk, M. H., van Paradijs, J., \& Zuiderwijk, E. J. 1995, A\&A, 303, 497

Wood, M. A. 1995, in Proc. Ninth European Workshop on White Dwarfs, ed.

D. Koester \& K. Werner (Berlin: Springer), 41 